\title{
Economic Evaluation of PET and PET/CT in Oncology: Evidence and Methodologic Approaches
}

\author{
Andreas K. Buck ${ }^{1}$, Ken Herrmann ${ }^{1}$, Tom Stargardt ${ }^{2,3}$, Tobias Dechow ${ }^{4}$, Bernd Joachim Krause ${ }^{1}$, and Jonas Schreyögg 2,3 \\ ${ }^{I}$ Nuklearmedizinische Klinik und Poliklinik, Klinikum rechts der Isar der Technischen Universität München, Munich, Germany; \\ ${ }^{2}$ Institute for Health Economics and Management, German Research Center for Environmental Health, Neuherberg, Germany; \\ ${ }^{3}$ Health Services Management, Munich School of Management, Ludwig-Maximilians-Universität, Munich, Germany; and ${ }^{4} I I I$. \\ Medizinische Klinik und Poliklinik, Klinikum rechts der Isar der Technischen Universität München, Munich, Germany
}

\begin{abstract}
Learning Objectives: On successful completion of this activity, participants should be able to (1) describe clinical PET/CT indications for which costeffectiveness has already been demonstrated; (2) recognize the need for future prospective studies to evaluate cost-effectiveness of PET/CT for multiple clinical indications; (3) apply suggested protocols of economic evaluation to future clinical trials assessing cost-effectiveness of PET/CT.

Financial Disclosure: The authors of this article have indicated no relevant relationships that could be perceived as a real or apparent conflict of interest.

CME Credit: SNM is accredited by the Accreditation Council for Continuing Medical Education (ACCME) to sponsor continuing education for physicians. SNM designates each JNM continuing education article for a maximum of 1.0 AMA PRA Category 1 Credit. Physicians should claim only credit commensurate with the extent of their participation in the activity.

For CE credit, participants can access this activity through the SNM Web site (http://www.snm.org/ce_online) through March 2011.
\end{abstract}

PET and PET/CT have changed the diagnostic algorithm in oncology. Health care systems worldwide have recently approved reimbursement for PET and PET/CT for staging of non-small cell lung cancer and differential diagnosis of solitary pulmonary nodules because PET and PET/CT have been found to be cost-effective for those uses. Additional indications that are covered by health care systems in the United States and several European countries include staging of gastrointestinal tract cancers, breast cancer, malignant lymphoma, melanoma, and head and neck cancers. Regarding these indications, diagnostic effectiveness and superiority over conventional imaging modalities have been shown, whereas cost-effectiveness has been demonstrated only in part. This article reports on the current knowledge of economic evaluations of PET and PET/CT in oncologic applications. Because more economic evaluations are needed for several clinical indications, we also report on the methodologies for conducting economic evaluations of diagnostic tests and suggest an approach toward the implementation of these tests in future clinical studies.

Key Words: positron emission tomography; computerized tomography; cost-effectiveness; image fusion; tumor staging; response to therapy; treatment individualization

J Nucl Med 2010; 51:401-412

DOI: 10.2967/jnumed.108.059584

Received Aug. 2, 2009; revision accepted Oct. 26, 2009.

For correspondence or reprints contact: Andreas K. Buck, Nuklearmedizinische Klinik und Poliklinik, Klinikum rechts der Isar der

TU München, Ismaninger Strasse 22, D-81675, München, Germany.

E-mail: andreas.buck@tum.de

This article is being published simultaneously in Journal of Nuclear Medicine Technology.

COPYRIGHT @ 2010 by the Society of Nuclear Medicine, Inc.
$\mathbf{T}$ he introduction of PET to clinical oncology in the early 1990s, and, more recently, combined imaging with CT, have substantially influenced the management of patients with cancer (1-6). The combination of a dedicated PET scanner and multislice helical CT enables integrated functional and high-resolution morphologic imaging (5). In many industrialized countries, the advantages of this diagnostic approach to differential diagnosis of undefined lesions, initial tumor staging, detection of relapse, and response monitoring have been recognized. Since its introduction to clinical medicine in 2001, PET/CT has represented one of the diagnostic modalities with the largest growth worldwide (Figs. 1 and 2) (5). In addition to the information derived from separate modalities, coregistration of PET with CT allows the locations of lesions seen on PET to be determined precisely. The addition of PET to CT data results in higher sensitivity and specificity of cancer imaging. Moreover, CT data can be used for attenuation correction of PET data, thereby reducing the scan duration by $20 \%-30 \%$. A standard examination covering the cervical region, chest, abdomen, pelvis, and thigh can be performed within 20-30 min.

In the United States, the Centers for Medicare and Medicaid Services allow reimbursement for most clinical indications in oncology, such as staging and restaging of lung cancer; esophageal, colorectal, and other gastrointestinal tract cancers; breast cancer; kidney and other genitourinary cancers; melanoma; head and neck cancers; 


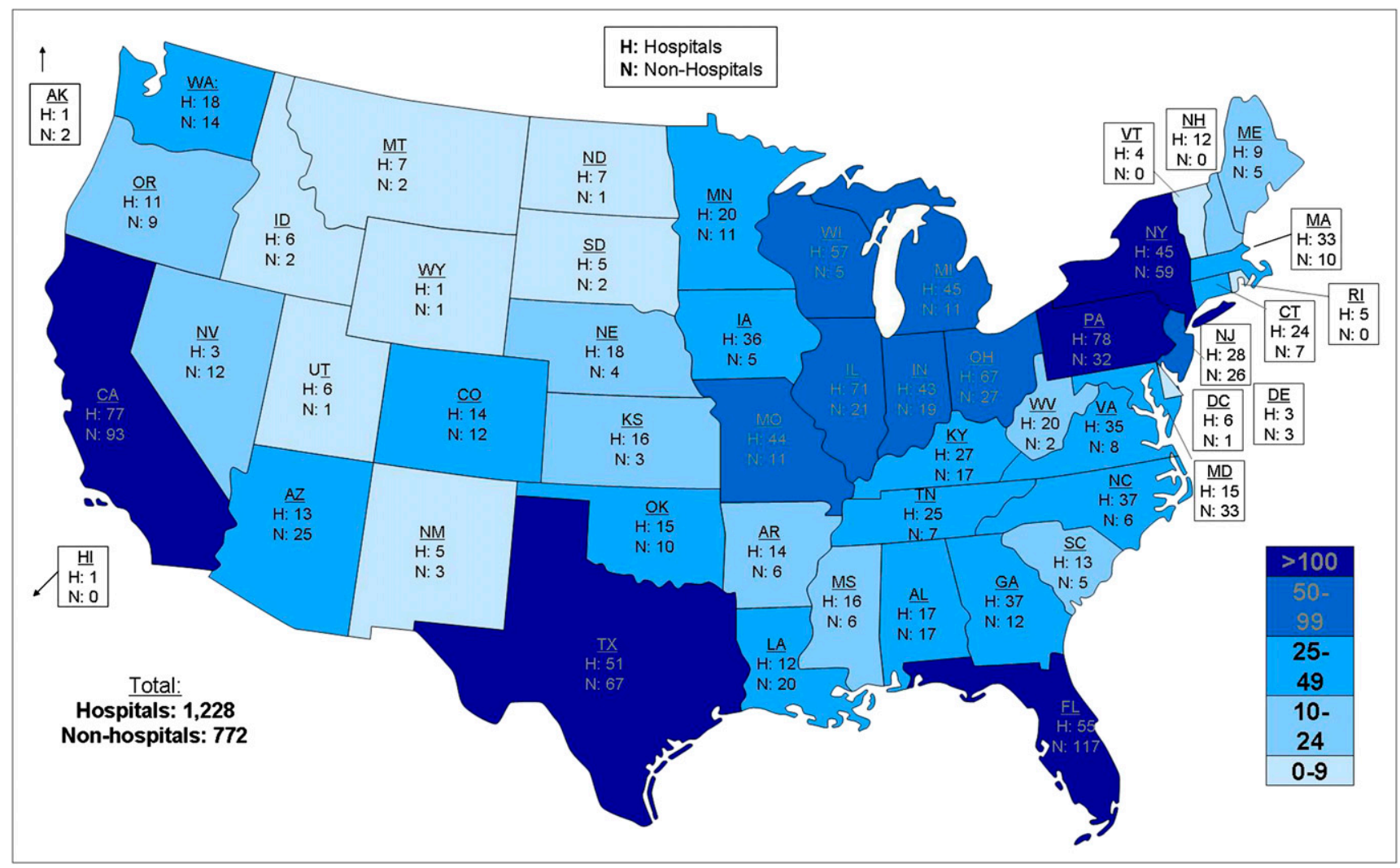

FIGURE 1. PET/CT represents one of the medical imaging modalities with the largest growth worldwide. In 2009, approximately 2,000 PET/CT scanners were installed in the United States and approximately 350 were installed in Europe. Considering a population of about 307 million in the United States and 830 million in Europe, the United States has installed about 6 times as many scanners as all of Europe but has only one third the population. (Courtesy of Siemens/CTI.)

and malignant lymphoma (Table 1). Assessment of response to treatment in breast cancer is also reimbursed. Recently, the Centers for Medicare and Medicaid Services have announced that further indications will be included in the list of services covered, provided that examinations are part of prospective clinical trials. In 2006, the National Oncologic PET Registry was launched to assess the influence of PET and PET/CT on care decisions (7-9). The registry is an attempt of the Centers for Medicare and Medicaid Services to further evaluate the clinical utility of PET and to make evidence-based decisions on coverage of PET for each cancer type or clinical indication. In Europe, the number of clinical indications for which reimbursement is approved varies between countries. In Germany, PET and PET/CT have recently been approved by the national health care system for management of non-small cell lung cancer (Table 1). Particularly, the use of PET/CT for initial tumor staging and for further characterization of solitary pulmonary nodules has been recognized as an adequate diagnostic test that is also cost-effective (10). At present, approximately 2,000 PET/CT scanners have been installed in the United States-about 6 times the number in all of Europe (approximately 350 PET/CT installations) (Fig. 1). Considering a population of 82 million in Germany, there are about 6.5 scanners per million people in the United States and 1.2 scanners per million people in Germany (Fig. 2).

Depending on the clinical needs, a variety of radiopharmaceuticals has been established for molecular or metabolic imaging with PET (6). The most relevant biomarker for functional characterization of cancers is the glucose analog ${ }^{18} \mathrm{~F}-\mathrm{FDG}$. Whereas conventional imaging modalities such as ultrasound, CT, or MRI allow detection of tumors based on characteristic morphologic alterations, PET enables the characterization of tumors based on molecular or metabolic alterations. After intravenous injection, ${ }^{18} \mathrm{~F}-\mathrm{FDG}$ is avidly taken up by tumor cells, similar to the native glucose molecule. After conversion of FDG to FDG-6-monophosphate by the cytosolic enzyme hexokinase, the metabolite cannot be further metabolized, leading to its metabolic trapping. Several radiopharmaceuticals that are capable of visualizing distinct pathophysiologic processes have been described. However, relevant cost-effectiveness studies have been performed exclusively for PET and PET/CT using the glucose analog ${ }^{18} \mathrm{~F}-\mathrm{FDG}$. 


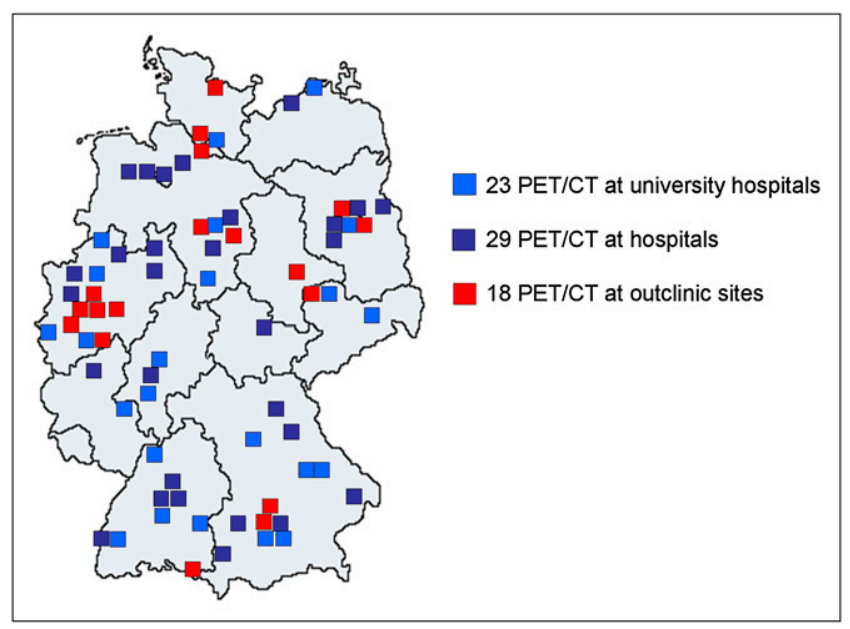

FIGURE 2. In Europe, introduction of PET/CT hybrid scanners has also led to an increase in their installations. Compared with the United States, however, a less accelerated growth has been observed. In 2009, 70 scanners were installed in Germany and 350 in all of Europe. (Courtesy of Siemens AG.)

\section{DIAGNOSTIC EFFECTIVENESS OF PET AND PET/CT IN ONCOLOGY}

\section{Tumor Detection and Differential Diagnosis of Benign and Malignant Tumors \\ ${ }^{18}$ F-FDG PET allows the detection of malignant tumors} based on increased glucose use. Tumors evident on morphologically based imaging modalities can be more specifically characterized. As an example, prospective studies indicated that for characterization of newly diagnosed solitary lung nodules, ${ }^{18} \mathrm{~F}-\mathrm{FDG}$ PET has a sensitivity of $89 \%-100 \%$, a specificity of $69 \%-100 \%$, and an accuracy of $89 \%-96 \%(11-13) .{ }^{18} \mathrm{~F}-\mathrm{FDG}$ is not tumor-specific and is also taken up by benign lesions such as inflammatory processes. A PET scan with negative findings can prevent surgical interventions at least in patients with an increased risk profile. PET and PET/CT can also be used to localize malignant tumors if conventional diagnostic procedures are not able to determine the primary site (cancer of unknown primary). In this regard, PET is especially helpful for localization of malignant primaries in the head and neck region. Also, in the case of increasing tumor markers or paraneoplastic syndromes, PET can help identify the primary tumor. A review of the diagnostic effectiveness of PET and PET/CT in oncology has recently been published by Fletcher et al. (14).

\section{Tumor Staging and Prognostic Stratification}

For planning the optimal treatment strategy, precise information about the initial tumor spread (tumor staging) is mandatory. If cancer is diagnosed at an early stage, the treatment of choice usually includes complete resection of the tumor, with curative intent. However, if the tumor has already reached distant organs, cure is usually not possible using surgery alone. In this situation, surgery has to be replaced or supplemented by systemic chemotherapy or radiotherapy to eliminate both the malignant primary and metastatic deposits or to prevent disease progression (Fig. 3). In this context, PET and PET/CT offer many advantages over conventional imaging modalities. Small tumor deposits in liver, lungs, bone, adrenal glands, or rare sites such as soft tissues, thyroid, or skin can be detected in a single examination. Micrometastases or solitary tumor cells, however, cannot be detected. Small lung metastases $(<6 \mathrm{~mm})$ can also be missed by PET, when performed as a separate imaging test. In principle, staging of all malignant tumors is possible. ${ }^{18} \mathrm{~F}$-FDG PET has a high accuracy for staging non-small cell lung cancer, gastrointestinal tract cancers (i.e., esophageal and colorectal cancer), malignant lymphoma, melanoma, thyroid cancer, and head and neck cancer. Depending on the tumor subtype, changes in therapeutic management of between $15 \%$ and $40 \%$ based on PET or PET/ CT findings have been described $(2,4,5,15,16)$. Some tumors, such as prostate cancer or neuroendocrine cancer, do not exhibit increased glucose use, leading to false-negative findings on ${ }^{18} \mathrm{~F}$-FDG PET. ${ }^{11} \mathrm{C}$-choline PET or ${ }^{11} \mathrm{C}$-choline $\mathrm{PET} / \mathrm{CT}$ has a high sensitivity and specificity for staging and especially restaging of prostate cancer. ${ }^{68} \mathrm{Ga}$-DOTATOC is a new PET tracer for imaging neuroendocrine cancers. A variety of molecular probes has been designed to address specific metabolic pathways. A clinical benefit regarding therapeutic management, increase of disease-free survival, and overall survival remains to be demonstrated for most of these radiotracers $(5,6)$. Also, cost-effectiveness analyses have not been performed so far.

Usually, the most important prognostic indicator of malignant tumors is the tumor stage at initial diagnosis. Risk stratification due to the TNM classification system, however, remains prone to error, since many patients who are diagnosed at an early stage will experience a relapse. Further factors such as tumor aggressiveness or metabolic activity of tumors could play an additional role in individual risk stratification and estimation of prognosis. A variety of studies have linked the intensity of ${ }^{18} \mathrm{~F}-\mathrm{FDG}$ and therefore glucose use in the primary tumor to progressionfree and overall survival. In lung cancer, for example, tumoral ${ }^{18}$ F-FDG uptake has been shown to represent an independent prognostic marker (17).

\section{Evaluation of Response to Treatment}

In individual patients, chemotherapy or radiation treatment shows varying therapeutic efficiency. A noninvasive method capable of identifying response to treatment on the individual level has therefore a high relevance. Using standard criteria (World Health Organization; Response Evaluation Criteria in Solid Tumors), response to treatment can be estimated from a significant reduction of the tumor volume. In contrast, PET allows detection of response earlier, when no reduction in tumor size can be measured. A reduction in glucose and, accordingly, ${ }^{18} \mathrm{~F}$-FDG metabolism is an in- 
TABLE 1. Reimbursement of PET and PET/CT in United States (59) and Germany (60)

\begin{tabular}{|c|c|c|c|c|}
\hline \multirow[b]{2}{*}{ Indication } & \multicolumn{2}{|c|}{ United States } & \multicolumn{2}{|c|}{ Germany } \\
\hline & $\begin{array}{c}\text { Initial } \\
\text { treatment } \\
\text { strategy }\end{array}$ & $\begin{array}{c}\text { Subsequent } \\
\text { treatment } \\
\text { strategy }\end{array}$ & $\begin{array}{l}\text { Initial } \\
\text { treatment } \\
\text { strategy }\end{array}$ & $\begin{array}{c}\text { Subsequent } \\
\text { treatment } \\
\text { strategy }\end{array}$ \\
\hline Head and neck cancer & C & C & - & - \\
\hline Esophagus cancer & C & C & - & - \\
\hline Gastric cancer & C & NOPR & - & - \\
\hline Small intestinal cancer & C & NOPR & - & - \\
\hline Colon and rectal cancer & C & C & - & - \\
\hline Anal cancer & C & NOPR $^{*}$ & - & - \\
\hline Hepatocellular carcinoma & C & NOPR & - & - \\
\hline Gallbladder and cholangiocellular carcinoma & C & NOPR & - & - \\
\hline Pancreatic cancer & C & NOPR & - & - \\
\hline Cancers of retroperitoneum and peritoneum & C & NOPR & - & - \\
\hline Non-small cell lung cancer & C & C & C & C \\
\hline Small cell lung cancer & C & NOPR & - & - \\
\hline Mesothelioma & C & NOPR & - & - \\
\hline Cancers of mediastinum; thymus carcinoma & C & NOPR & - & - \\
\hline Sarcoma of bone & C & NOPR & - & - \\
\hline Soft-tissue sarcoma & C & NOPR & - & - \\
\hline Melanoma & $\mathrm{C} / \mathrm{-}^{\dagger}$ & C & - & - \\
\hline Skin cancers (nonmelanoma) & C & NOPR & - & - \\
\hline Breast cancer & $\mathrm{C} /$ †‡ $^{\dagger+}$ & C & - & - \\
\hline Uterine cancer & C & NOPR & - & - \\
\hline Cervix carcinoma & C/NOPR $\$$ & C & - & - \\
\hline Ovarian cancer & C & C & - & - \\
\hline Prostate cancer & - & NOPR & - & - \\
\hline Bladder cancer & C & NOPR & - & - \\
\hline Kidney and other urinary tract cancers & C & NOPR & - & - \\
\hline Primary brain tumors & C & NOPR & - & - \\
\hline Thyroid cancer & C & C/NOPR\| & - & - \\
\hline Other endocrine tumors & C & NOPR & - & - \\
\hline Cancer of unknown primary & $\mathrm{C}$ & NOPR & - & - \\
\hline Lymphoma & C & C & - & - \\
\hline Myeloma & C & C & - & - \\
\hline Leukemia & NOPR & NOPR & - & - \\
\hline Neuroendocrine tumors & C & NOPR & - & - \\
\hline Other cancers & C & NOPR & - & - \\
\hline
\end{tabular}

"Some Medicare contractors include anal cancer in their local coverage of "colorectal cancer"; for PET facilities served by those carriers, PET for subsequent treatment evaluation of anal cancer would be a covered indication.

${ }^{\dagger} \mathrm{PET}$ is not covered for initial staging of axillary lymph nodes in patients with breast cancer and of regional lymph nodes in patients with melanoma but is covered for detection of distant metastatic disease in high-risk patients with breast cancer or melanoma.

"PET is not covered for "diagnosis" of breast cancer to evaluate suggestive breast mass. However, PET is covered for initial treatment-strategy evaluation of patient with axillary nodal metastasis of unknown primary origin or patient with paraneoplastic syndrome potentially caused by occult breast cancer.

SPatient must have prior CT or MRI negative for extrapelvic metastatic disease for PET to qualify as covered indication for initial treatment-strategy evaluation. Patients who do not qualify for this covered indication (e.g., because CT or MRI was not done or because either CT or MRI showed extrapelvic metastatic disease) can be entered on NOPR.

"To qualify as covered indication for subsequent treatment-strategy evaluation, thyroid cancer must be of follicular cell origin and have been previously treated by thyroidectomy and radioiodine ablation and patient must have serum thyroglobulin level $>10 \mathrm{ng} / \mathrm{mL}$ and negative whole-body ${ }^{131}$ I findings. Patients who do not qualify for this covered indication (e.g., because tumor is not of follicular cell origin, thyroglobulin is not elevated, or ${ }^{131}$ I whole-body imaging was not performed or is positive) can be entered on NOPR.

$\mathrm{C}=$ covered (not eligible for entry in National Oncologic PET Registry [NOPR]); NOPR = covered only with entry in NOPR; $-=$ not covered nationally (not eligible for entry in NOPR).

Modified from http://www.cancerpetregistry.org/indications_facilities.htm.

dicator of effective therapy and has prognostic utility regarding the effectiveness of additional cycles of therapy $(18,19)$. In the case of a nonresponse, a combination of cytostatic drugs can be applied, the radiation dose can be changed, or the entire therapeutic regime can be changed. In breast cancer, rapid reduction of ${ }^{18} \mathrm{~F}-\mathrm{FDG}$ uptake has been demonstrated as soon as after 1 cycle of chemotherapy, whereas in nonresponders a stable or even increased ${ }^{18} \mathrm{~F}$ - 


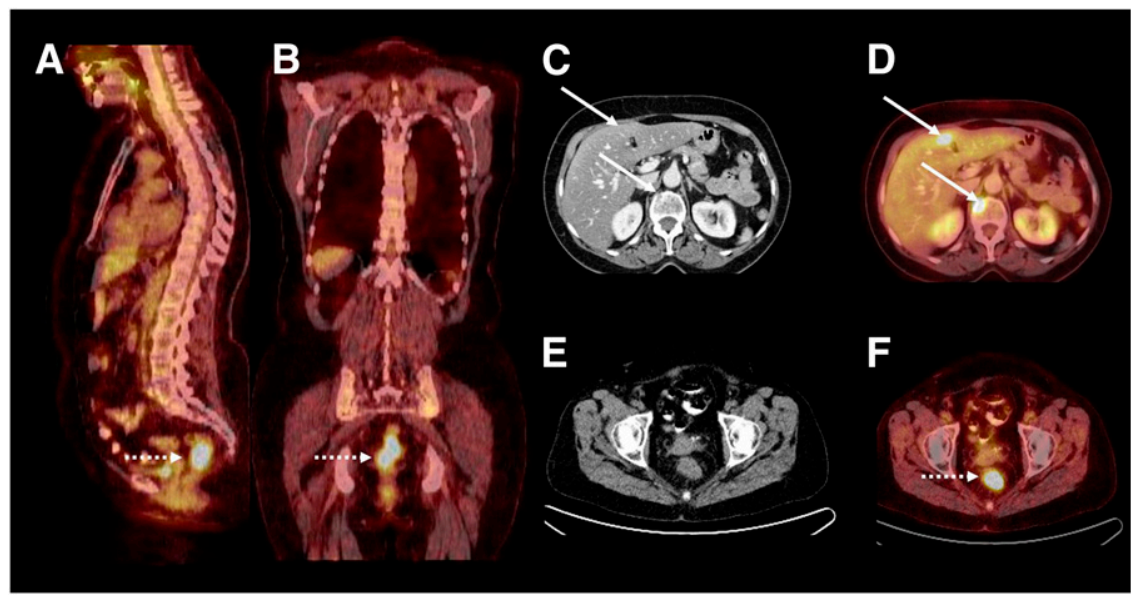

FIGURE 3. PET/CT has greater diagnostic accuracy than separately performed imaging modalities. In this patient at initial diagnosis of colorectal cancer, coronal (A) and sagittal (B) PET/ $\mathrm{CT}$ images indicate increased metabolic activity of malignant primary (arrows); transaxial CT (C) and PET/CT (D) images indicate synchronous bone and liver metastases (arrows), leading to change from curative resection to systemic chemotherapy; and transaxial CT (E) and PET/CT (F) images at another level indicate primary tumor.

FDG uptake has been demonstrated. A variety of tumor entities such as malignant lymphoma, gastric and esophageal cancer, head and neck cancer, and lung cancer has also shown significant reduction of the ${ }^{18} \mathrm{~F}-\mathrm{FDG}$ uptake in tumors responding to therapy. In responders, a significantly longer disease-free and overall survival has been demonstrated (19). However, we lack prospective studies demonstrating a clinical benefit of PET-based alterations of therapeutic management. Recently, the team of Lordick demonstrated that patients with tumors of the esophagogastric junction and PET response have a good long-term prognosis. In PET nonresponders, treatment was discontinued and resective surgery followed without additional cycles of therapy. Compared with a historic collective receiving 6 cycles of chemotherapy despite PET nonresponse (20), nonresponders have shown a tendency toward better median survival (21). However, randomized clinical trials are necessary to prove a positive influence of PET on therapeutic management and on the course of disease.

\section{Restaging and Detection of Recurrent Cancer}

After a definite surgical intervention or chemo- or radiotherapy has been performed, imaging modalities and diagnostic tests are mandatory for early diagnosis of tumor recurrence emerging from remaining tumor cells. In daily clinical practice, differentiation between scar tissue and residual vital tumor is a frequent problem. On morphologically based imaging modalities, both structures may appear as a nonclassifiable tissue formation. Subsequently, an invasive test such as fine-needle aspiration cytology or core biopsy is often necessary to provide further differential diagnosis. The differentiation of scar tissue from vital residual tumor is a prerequisite of functional imaging with PET and PET/CT. Whereas tumor recurrence is associated with increased glucose metabolism and, hence, increased ${ }^{18} \mathrm{~F}-\mathrm{FDG}$ uptake, scar tissue usually presents with absence of or mild ${ }^{18} \mathrm{~F}-\mathrm{FDG}$ uptake, compared with surrounding normal tissue. PET and PET/CT are especially effective for surveillance of colorectal, esophageal, lung, and breast cancer; melanoma; head and neck cancer; malignant lymphoma; and brain tumors $(1,2,4)$. The clinical benefit of PET and PET/CT for restaging patients with differentiated thyroid cancer and an increase in the tumor marker thyroglobulin has also been demonstrated.

\section{Radiation Treatment Planning}

The use of metabolic information for planning the radiation field leads to biologic target volumes, which can alter the radiation field by increasing or reducing the target volume. The additional identification of tumor deposits not detected by other imaging modalities leads to an increase in the radiation field. On the other hand, the radiation field can be reduced if nonmalignant alterations such as atelectatic lung tissue can reliably be identified as benign. Consequently, the radiation dose to neighboring structures can be reduced. It has been reported that the inclusion of PET data leads to an alteration of the radiation field in up to $60 \%$ of patients $(22,23)$. PET-based radiation treatment planning, however, is not trivial. Especially, delineation of the primary tumor is subject to a relevant interobserver variability. We lack standardized evaluation criteria for PET that exceed simple visual interpretation and allow quantification of metabolic processes. The introduction of PET/CT hybrid scanners has reduced errors originating from image fusion. Several prospective clinical trials have demonstrated that overall survival of patients receiving PET-based radiation treatment planning was significantly higher than that of patients treated without the use of PET (24). However, prospective, randomized trials have to be performed to demonstrate if the implementation of PET also enhances disease-free and overall survival.

\section{Role of PET for Development of New Anticancer Drugs}

PET offers unique characteristics that can be used for the development of new anticancer drugs $(1,6)$. The therapeutic effect of innovative drugs can be measured noninvasively using specific biologic endpoints such as tumor cell proliferation ( $3^{\prime}$-deoxy- $3^{\prime}-{ }^{18} \mathrm{~F}$-fluorothymidine), glucose metabolism $\left({ }^{18} \mathrm{~F}-\mathrm{FDG}\right)$, tumor perfusion $\left({ }^{15} \mathrm{O}-\mathrm{H}_{2} \mathrm{O}\right)$, protein biosynthesis $\left(O-\left(2-{ }^{18} \mathrm{~F}-\right.\right.$ fluoroethyl $)$-L-tyrosine, methyl-L- ${ }^{11} \mathrm{C}-$ methionine), or inhibition of neoangiogenesis (radiolabeled 
peptides specifically binding to the integrin $\alpha_{v} \beta_{3}$ such as ${ }^{18} \mathrm{~F}-$ galacto-RDG containing the amino acid sequence arginine, glycine, and aspartic acid-RGD in the single-letter code). Overexpression of target structures such as thymidylate synthase, vascular endothelial growth factor receptor, erbB2, or estrogen receptors can be specifically detected with ${ }^{11} \mathrm{C}$ thymidine, radiolabeled antibodies, or ligands binding to vascular endothelial growth factor receptor, erbB2, or estrogen receptors $\left({ }^{18} \mathrm{~F}\right.$-fluoro- $17-\beta$-estradiol). The evaluation of biologic endpoints also allows one to provide evidence of a proposed mechanism of action. PET can also be used for specific measurement or assessment of the expression of reporter genes. For example, the substrate ${ }^{124} \mathrm{I}$-fluoro-5-iodo$1-\beta$-D-arabinofuranosyluracil can be used for quantification of viral thymidine kinase expression, and $\mathrm{Na}^{124} \mathrm{I}$ is a suitable biomarker for detection of natrium iodide symporter expression.

Generic endpoints can also be studied with PET. Drugs and biochemical probes including small molecules, proteins, or antibodies can be labeled with positron emitters. So far, a large number of drugs have been radiolabeled for PET, including nitrosourea, fluorouracil, tamoxifen, cisplatin, and, more recently, gefitinib, imatinib, and others. The pharmacokinetics of a specific drug in tumor and normal tissues can be evaluated in animal studies or in clinical phase I or II trials.

\section{COSTS FOR PET AND PET/CT}

On a patient basis, costs for PET and PET/CT are decreasing with the increasing numbers of examinations performed. In Germany, for example, costs per examination range between approximately $€ 600$ ( $\$ 885)$ and $€ 1,000(\$ 1,474)$; the amount for production and delivery of radiopharmaceuticals is approximately $€ 180-€ 260$ (\$265-\$383) per scan. In a recent survey in Great Britain, costs of $£ 635-£ 1,300$ ( $\$ 1,030$ $\$ 2,109$ ) were mentioned for PET (25). In Europe, reimbursement for PET and PET/CT examinations varies significantly depending on the respective health care systems. In the United States, reimbursement for PET and PET/CT is provided by the Medicare program. For examinations performed on inpatients or at hospital outpatient departments, a median amount of $\$ 952.83$ is reimbursed. The amount consists of $\$ 855.43$ for the examination and $\$ 97.40$ for the analysis. Under certain conditions, additional costs for the production of radiopharmaceuticals are reimbursed.

\section{METHODS FOR ECONOMIC EVALUATION}

In a world of limited financial resources, those who make reimbursement decisions increasingly require an assessment of economic benefit to further demonstrate that diagnostic procedures or interventions are able to contribute. The socalled economic evaluations go beyond pure effectiveness measurements by combining costs and consequences (outcomes) of defined diagnostic procedures or interventions. Commonly, there are 3 approaches toward economic evalu- ation, with the type of outcome measurement determining the approach (26): cost-effectiveness analysis (CEA), costutility analysis (CUA), and cost-benefit analysis (CBA).

\section{CEA}

CEA compares alternative interventions using costs and a common effectiveness measure (e.g., correct staging or life-years gained). The results of such comparisons may be stated either in terms of costs per unit of effect (e.g., costs per life-years gained) or effects per unit (life-years gained per dollar spent). In this context, the relative cost-effectiveness of alternative tests can be assessed as long as the alternatives under consideration are not of exceptionally different scale. To compare alternatives, the incremental cost-effectiveness ratio (ICER) is calculated. It shows the additional costs caused by the implementation of a new diagnostic test or intervention and relates them to the health outcome $($ ICER $)=\left(\right.$ costs $_{\text {new test }}-$ costs $\left._{\text {standard test }}\right) /($ lifeyears gained $_{\text {new test }}-$ life-years gained $\left._{\text {standard test }}\right)$. Acceptable ICER thresholds (maximum ICERs) for reimbursement differ between countries according to wealth and societal preferences. For instance, the English National Institute for Health and Clinical Excellence defines a threshold of $£ 30,000(\sim 49,000)$ per additional life-year gained as acceptable (27). However, in reality, other features such as the innovative nature of a therapy are also considered. The advantage of CEA is its simplicity. Study costs are usually lower than for CUA and CBA, because effectiveness is usually measured in daily routine care or in clinical trials whereas CUA and CBA require interviewers guiding patients to answer defined questions.

\section{CUA}

CUA compares interventions using costs and outcomes that are adjusted for quality of life, such as quality-adjusted life-years (QALYs) or disability-adjusted life-years. Utilities usually require interviewers who guide patients to answer specific questions. Utilities can be generated either by measuring preferences for health outcomes, that is, asking patients about their preference for one state of health over another (e.g., time trade-off or standard-gamble methods), or by using a multiattribute health status classification system (e.g., quality-of-well-being scale or EQ-5D). Results are usually stated as costs per QALY. CUA has 2 advantages over CEA. First, CUA allows for quality-of-life adjustments. Second, whereas effectiveness measurements as part of CEA are often limited to indications or treatment areas (e.g., the parameter "correctly staged patients" is limited to diagnostic tests), CUA provides generic outcome measures to compare different programs, facilitating comparisons between alternative programs. The problem with qualityof-life measurement is that there are numerous methods to measure utilities, and results may vary according to the method used. Preferences of evaluation agencies around the world regarding methods for measurement of the quality of life still differ, and a gold standard remains to be determined. 
CBA measures not only costs but also the consequences in monetary units. Diagnostic procedures or interventions are commonly adopted if the monetary benefits exceed procedure-related costs. In this situation, the adoption results in a net benefit. Thus, this approach "theoretically" does not require a comparison to other diagnostic procedures or interventions. However, in reality, there is a fixed health care budget that allows the adoption of only a fixed number of diagnostic procedures or interventions, and those with the highest net benefit are selected. Similar to CUA, benefit is measured by asking patients for their preferences. Again, there are many different methods to measure benefits, such as contingent valuation studies or revealed preference studies. However, CBA is still in its experimental stage, and gold standards for how this analysis should be performed remain to be determined. In addition, benefit measurement is quite problematic from an ethical point of view as it implies a valuation of different lives. For these reasons, evaluation agencies usually prefer CEA and CUA.

\section{COST-EFFECTIVENESS OF PET AND PET/CT IN SELECTED CANCERS}

The number of studies reporting on economic evaluations of PET and PET/CT is limited. Compared with other widespread imaging modalities such as CT and MRI, however, the number of available publications is higher. Presumably, this originates from the general assumption that PET and PET/CT represent costly diagnostic tests. Accordingly, economic evaluations were requested early in the clinical implementation of PET. Available studies almost exclusively represent cost-effectiveness studies. von Schulthess et al. announced a high potential for PET/CT as a cost-effective approach for diagnostic management of cancer (5). In most cancers, prospective studies lack evaluation of the costeffectiveness of PET/CT. Moreover, clinical scenarios in which this modality can be implemented cost-effectively have not been defined yet. Initial studies have shown at least mild improvement of diagnostic accuracy, compared with separately performed CT and PET studies. Therefore, results obtained for PET can generally be extended to PET/CT.

\section{DIFFERENTIAL DIAGNOSIS OF SOLITARY PULMONARY NODULES}

Differentiation of benign from malignant pulmonary nodules represents the first clinical indication for which cost-effectiveness of PET has been demonstrated. The potential of PET to be implemented cost-effectively in the diagnostic work-up can be explained by a higher specificity and higher diagnostic accuracy than is available with the standard imaging approach. Costs for fine-needle biopsy and thoracotomy can be prevented if a suggestive lesion can be reliably classified as benign. In a casetracking study, Valk et al. reported that the use of PET has led to a reduction of costs of $\$ 2,200$ per patient (28). A second research group in the United States reported cost savings of $\$ 91-\$ 2,200$ per patient if the standard diagnostic algorithm consisting of CT only is supplemented by PET (CT + PET strategy) (29). This finding is evident for a large pretest likelihood of $0.12-0.69$. Two Australian research groups reported similar results. They published cost savings of AU\$505 (\$459) and AU\$935 (\$849) (30) or AU\$774 (\$703) (31) per patient, if PET was performed additionally to CT. In contrast, a Japanese group reported additional costs for a CT + PET strategy per life-year saved (ICER $=\$ 1,557)$ (32). In Germany, Dietlein et al. also reported additional costs for the PET-based strategy and an ICER of $€ 3,218$ (\$4,745) per life-year saved (33). All authors concluded that the additional costs for PET are in an acceptable range.

Despite a varying prevalence of malignant pulmonary nodules in individual studies, PET has been shown to be cost-effective in a variety of geographic regions. CT-based strategies seem to be cost-effective only if the patient collective has a high pretest likelihood for malignant nodules. Cost-effectiveness of PET regarding the differential diagnosis of undefined pulmonary nodules can be derived from the fact that PET frequently shows additional findings such as secondary cancers or metastatic tumor deposits causing a change in clinical management and offering further potential for cost savings, that is, omission of resective surgery performed with curative intent.

\section{Non-Small Cell Lung Cancer}

The cost-effectiveness of ${ }^{18} \mathrm{~F}$-FDG PET in staging lung cancer is based on the potential of PET to detect metastases that cannot be diagnosed using standard tests. Non- ${ }^{18} \mathrm{~F}-$ FDG-avid pulmonary lesions most likely represent benign lesions. Therapeutic management will change in 19\%-41\% of patients if PET is added to the diagnostic algorithm $(11,34)$. In $10 \%-14 \%$ of patients, detection of distant metastases has prevented surgical interventions performed with curative intent (15). A randomized study showed that the number of "futile" thoracotomies was significantly reduced by the addition of ${ }^{18} \mathrm{~F}$-FDG PET to the diagnostic algorithm: 19 of 82 patients who underwent PET had a futile thoracotomy, compared with 29 of 96 who did not undergo PET (35).

Cost-effectiveness of ${ }^{18} \mathrm{~F}$-FDG PET has been shown in 2 independent studies $(33,36)$. Both groups demonstrated that ${ }^{18} \mathrm{~F}$-FDG PET is cost-effective if it detects mediastinal lymph node metastases that were not evident on CT. This is the case in up to $20 \%$ of patients presenting with normal CT findings but ${ }^{18} \mathrm{~F}-\mathrm{FDG}$-positive nodes indicating mediastinal lymph node metastases. Accordingly, a significantly lower number of patients underwent curative resective surgery. Conversely, patients with mediastinal nodes suggestive on CT but negative on PET do not have to undergo additional mediastinoscopy. These patients can directly undergo resective surgery with curative intent. However, in the case of ${ }^{18} \mathrm{~F}-\mathrm{FDG}$-positive nodes, mediastinoscopy is recommended to rule out falsepositive findings (37). Because of the additional costs of the PET scan, the contribution of PET to cost savings is not 
significant in the case of ${ }^{18} \mathrm{~F}$-FDG-positive mediastinal nodes. Overall, the use of ${ }^{18} \mathrm{~F}-\mathrm{FDG}$ PET for staging and restaging non-small cell lung cancer has been shown to be cost-effective and has been approved recently by the German health care system (Federal Joint Committee, G-BA) (10).

\section{Colorectal Cancer}

The conventional diagnostic algorithm performed for imaging recurrent colorectal cancer (i.e., ultrasound of the liver, CT, MRI) has several limitations. As a consequence of imprecise restaging, only $30 \%-40 \%$ of liver resections are performed with curative intent, causing a relatively high number of palliative surgical interventions and therefore unnecessary costs. The ratio of additional costs for a PETbased imaging algorithm and the saving of additional lifeyears based on decision models has been evaluated in only a few studies. For the assessment of cost-effectiveness, 2 studies considered the reimbursement policy of the U.S. health care system $(28,38)$, whereas a third study considered the regulations of the French health care system (39). In patients with recurrent colorectal cancer and suspicion of hepatic metastases, Gambhir et al. compared various diagnostic algorithms, including a combination of the tumor marker carcinoembryonic antigen and CT scan (carcinoembryonic antigen $+\mathrm{CT}$ ) or the additional use of PET (carcinoembryonic antigen $+\mathrm{CT}+\mathrm{PET})(40)$. Calculations were based on decision models and revealed cost savings of $\$ 220$ per patient in the carcinoembryonic antigen + CT + PET group and a gain of life expectancy of $2 \mathrm{~d}$, compared with the conventional approach (carcinoembryonic antigen $+\mathrm{CT}$ ). Using a similar approach, Park et al. compared the diagnostic algorithms CT only and additional implementation of PET $(\mathrm{CT}+\mathrm{PET})$ in patients with suspicion of recurrent colorectal cancer, as indicated by rising tumor markers (carcinoembryonic antigen $>5 \mathrm{ng} / \mathrm{mL}$ ) (38). According to the literature, unnecessary surgical interventions can be prevented by ${ }^{18}$ F-FDG PET in $2.8 \%$ of patients presenting with rising tumor marker carcinoembryonic antigen after definite treatment for colorectal cancer. Transferring these data to the incidence of recurrent colorectal cancer in the United States, we find that a total of 6,000 PET examinations are associated with 167 surgical interventions cancelled because of the findings at PET. In contrast to the results of Gambhir et al., implementation of PET in the diagnostic algorithm increased costs by $\$ 429$ per patient but also increased life expectancy by $9.5 \mathrm{~d}$. The ICER is as high as $\$ 16,437$ per life-year saved. Lejeune et al. reported a cost analysis based on survival data of the "Registre Bourgignon des Cancers Digestif" (39). In contrast to the other studies mentioned, suspicion of relapse was based on ultrasound imaging of the abdomen. Calculations were again based on decision models and revealed cost savings of $\$ 3,213$ per patient if PET was implemented in the diagnostic work-up (CT + PET). In this study, PET was cost-effective because of a marked reduction in the number of surgical interventions (by 88\%), compared with the CT-based approach. On the other hand, a gain of life expectancy could not be demonstrated.

\section{Head and Neck Cancers}

Two studies performed in the United States could demonstrate the cost-effectiveness of PET in head and neck cancers. Valk et al. prospectively evaluated patients with resectable head and neck cancer or local tumor recurrence (28). Detection of distant metastases led to cancellation of curative surgery and resulted in a cost saving of $\$ 500$ per patient. Another study analyzed the cost-effectiveness of PET in patients with squamous cell carcinoma of the head and neck and absence of clinical signs indicating locoregional lymphatic spread on CT (41). In this patient collective, PET is cost-effective if lymph node metastases can be detected more sensitively than with the standard imaging approach. By preventing morbidity and enhancing the overall survival and quality of life, PET-based tumor staging was associated with an increase of median survival of $0.13 \mathrm{y}$ or 0.44 QALYs. PET resulted in additional costs of $\$ 1,107$, taking into account the ICER of $\$ 871$ per life-year saved or $\$ 2,505 / \mathrm{Q} A L Y$. Given the prevalence of ${ }^{18} \mathrm{~F}-\mathrm{FDG}-$ positive lymph node metastases of $16 \%-36 \%$, costs were below the approved threshold of $\$ 50,000$.

\section{Malignant Lymphoma}

Cost-effectiveness of PET and PET/CT regarding staging of Hodgkin disease and non-Hodgkin lymphoma has been evaluated by several groups in the United States and Germany. In the study by Hoh et al., the addition of PET to the diagnostic work-up increased the diagnostic accuracy from $83 \%$ to $94 \%$ and reduced costs for tumor staging by approximately $\$ 1,685$ (42). A similar finding regarding the impact of PET on tumor staging has been published by Klose et al. The authors reported an increase of diagnostic accuracy from $82 \%$ to $100 \%$. However, the addition of PET increased the overall costs for tumor staging (43). Costs for each correctly diagnosed tumor stage were €478 (\$704) using a CT-based approach and $€ 3,133$ (\$4,613) using a PET-based approach. For a patient correctly staged by PET but falsely staged by CT, additional costs of $€ 15,065$ $(\$ 22,182)$ were calculated. Accordingly, an economic advantage for PET could not be demonstrated in that series.

\section{Pancreatic Cancer}

A single study reports on the cost-effectiveness of PET/ CT for staging of pancreatic cancer (44). In 59 patients, the positive predictive value was $91 \%$ and the negative predictive value was $64 \%$. PET was able to identify additional metastases in 5 patients and secondary malignancies in a further 2 patients. Consequently, the therapeutic management was changed in $16 \%$ of patients. An amount of $\$ 37,700$ was calculated for the surgical procedure, and a daily rate of $\$ 1,200$ for the hospital stay. Cost for PET/CT was $\$ 1,925$ per examination (cost for production and delivery of the ${ }^{18} \mathrm{~F}-\mathrm{FDG}$ was $\$ 425$; cost for the examination with PET/CT was $\$ 1,500)$. For histologic confirmation of 
PET/CT findings, an additional cost of $\$ 12,010$ was calculated (i.e., CT-guided fine-needle aspiration cytology and thoracoscopic wedge resection). Because of a change from tumor resection $(-\$ 188,500)$ to palliative chemotherapy, \$1,066 was saved per patient (total amount, \$62,912), taking into account the added costs for PET/CT and diagnostic procedures arising from PET/CT results.

\section{Other Solid Neoplasms}

Analyses of cost-effectiveness in a multitude of other tumor entities have been evaluated predominantly in singlecenter studies having few patients. In a cohort consisting of 45 patients with recurrent melanoma, PET was able to detect previously unknown distant metastases in $27 \%$ of patients or could assign suggestive lesions as benign (28). Curative surgical intervention was cancelled, resulting in cost saving of $\$ 2,175$ per patient. Cost-effectiveness of the PET strategy regarding detection of locoregional lymph node metastases from breast cancer has been studied in Australia and the United States. The American study reported a reduction of costs by $\$ 2,300$ (45), and the Australian study by AU\$550 (\$499) (30). However, the clinical relevance of PET or PET/CT regarding staging of the axilla is now reduced because of implementation of the sentinel lymph node concept.

\section{SUGGESTED SETUP FOR ECONOMIC EVALUATION OF PET/CT}

\section{Choosing a Type of Economic Evaluation}

The main goal of economic evaluation is to compare the costs and consequences of alternative options (26). Although CEA allows a comparison of the costs and effectiveness of alternatives (e.g., costs per life-year saved), CUA is superior in that health-related quality-of-life (HRQL) measures combine differences in life-years saved with differences in toxicity profiles and adverse events $(46,47)$. A CUA is even more important when differences in clinically relevant outcomes between interventions are expected to be small. In addition, CUA allows direct comparison for a broad range of interventions, whereas a CEA can compare only interventions for which the same outcome measures are essential (46). To compare the effectiveness of diagnostic methods in oncologic applications, a CEA would be sufficient. However, if the results are to be used in a broader context, as in a decision to allocate resources between different health care programs by regulatory agencies such as the National Institute for Health and Clinical Excellence in England or the Institute for Quality and Efficiency in Health Care in Germany, the additional use of CUA is recommended.

\section{Study Design}

Because PET, CT, and PET/CT are diagnostic methods used for further work-up of patients with predominantly lifethreatening diseases, it would be unethical to do a fully randomized, controlled trial in which treatment choice is based on only 1 of the 3 diagnostic methods to which patients are randomly allocated (48). Also, there is evidence that PET is superior in staging tumors, compared with CT $(33,43,49)$. Withholding available diagnostic results to patients in the CT group of a randomized controlled trial might result in increased mortality and thus be clearly inappropriate $(48,50)$.

Instead, each cancer patient should be diagnosed with all 3 methods. For each diagnostic method, the results should then be allocated anonymously to external experts for clinical or scientific evaluation. Although the actual treatment choice for the patient is based on all available diagnostic results, treatment choice within the economic evaluation should be based on the staging according to each method. Thus, in the economic evaluation it is possible that the patient who has in fact received surgical treatment is considered nonresectable according to CT, resectable according to PET, and nonresectable according to PET/CT. We also recommend that, in the economic evaluation, the patient is assumed to be treated strictly according to guidelines.

\section{Measurement of Treatment Costs}

To calculate CEA and CUA, data on costs must be obtained. From an economic perspective, the data are differentiated between costs incurred for the treatment of the patient (so-called direct medical costs; e.g., hospital costs or cost of pharmaceuticals) and other costs incurred because of the illness but not related to the treatment itself (so-called indirect costs; e.g., loss of productivity due to absence from work). When calculating direct costs in economic evaluations of diagnostic tests, one should keep in mind the costs for potential treatment (e.g., surgery or chemotherapy, following the recommendations of the diagnostic tests) in addition to the costs for the diagnostic tests.

The decision on which resources consumed in treating a disease are considered "costs" in an economic evaluation also depends on the perspective chosen for the economic analysis. Although, from the payer's perspective, indirect costs are usually irrelevant because they are not borne by the payer, indirect costs are highly relevant from the societal perspective. Also, the amount of costs incurred may differ depending on the perspective chosen. Although the costs of treatment from the provider's perspective are the production costs of services, the costs of treatment from the payer's perspective are equal to the reimbursement for the services. The choice of perspective depends on the group that is to be addressed by the study. For example, when the study is to provide information for a reimbursement decision by agencies such as the National Institute for Health and Clinical Excellence and the Institute for Quality and Efficiency in Health Care, it is recommended that the payer's perspective be used $(27,51)$.

\section{Measurement of Effectiveness}

It is important that the effectiveness measures used in an economic evaluation are generally accepted and meaningful. Although effectiveness can always be defined in great detail from a medical perspective (e.g., stages for the classification of cancer), it might be important to apply a broader definition in an economic evaluation. In the case of correct 
staging as a measure of the effectiveness of CT, PET, or PET/ CT, the more detailed the stages, the larger the study population needed to produce meaningful results.

\section{Utility Measurement}

Utility measurement in economic evaluations, as well as the availability of validated instruments to measure utility, has greatly increased. For reasons of feasibility and costs, researchers often prefer multiattribute health status classification systems (also called HRQL instruments) over instruments to measure preferences. The basic idea of HRQL instruments is to let the patient or-depending on the illness that is evaluated - a relative (as a proxy), or a clinician, rate the patient's HRQL on a questionnaire (52). The questionnaires to measure HRQL usually consist of multiple items related to the impact of the disease on the physical or functional status of the patient or global scores. For non-small cell lung cancer, there are disease-specific HRQL instruments that can be transferred into QALYs, such as the Lung Cancer Symptom Scale $(52,53)$. Also, multiple general (not disease-specific) HRQL instruments, such as the EQ-5D/visual analog scale (54), have been used (47,55).

Although direct HRQL measurement in a clinical study would be preferred over alternatives, it is sometimes impractical. For the comparison of diagnostic methods such as CT, PET, and PET/CT for cancers, multiple HRQL measurements would be necessary during follow-up (e.g., 2, 4, and $6 \mathrm{wk}$ after surgery). Given that data for the evaluation of the effectiveness of CT, PET, and PET/CT are obtained primarily during the initial hospital visit, HRQL measurements conducted at regular intervals would greatly increase study efforts and costs. If researchers are faced with heavy budget constraints, we recommend that they derive HRQL from the literature or make reasonable assumptions if direct HRQL measurement is not possible. We also recommend that they conduct sensitivity analysis of assumptions to analyze the impact on the results.

For example, in a study on PET versus CT for non-small cell lung cancer by the Centre for Health Economics Research and Evaluation at the University of Technology Sydney, quality of life was defined to be 1 for patients who were alive and 0 for patients who had died. To account for surgical morbidity, a loss in quality of life of 0.15 QALYs was assumed for patients who underwent surgery, based on the results of similar studies. In the sensitivity analysis, the surgical morbidity rate was varied in order to test its impact on the results (56). In any case, HRQL estimations should consider potentially different scenarios after the diagnostic test, such as whether surgery is performed or not.

\section{Discounting of Costs and Benefits}

When costs or benefits are incurred over time, their discounting is necessary in order to reflect time preferences. In general, time preferences are expected to be positive; that is, patients prefer benefits sooner rather than later (57). Thus, future costs or benefits are divided by $(1+\mathrm{d})^{\mathrm{t}}$, where $\mathrm{d}$ is the discount rate per period and $\mathrm{t}$ is the number of the time period. Although the National Institute of Clinical Excellence considers $3.5 \%$ to be an appropriate annual discount rate for cost and health benefits (27), the Institute for Quality and Efficiency in Health Care recommends using $3 \%$ for costs and benefits but is also willing to accept a lower or zero discount rate for health benefits if differential timing has already been incorporated into the measurement of benefits (51). Both regulatory agencies insist on a sensitivity analysis using different discount rates $(27,51)$.

With regard to preventive and other programs characterized by early investment and late health outcome, such as diagnostic methods, it can be argued that discounting of benefits discriminates programs characterized by early investment and late health outcome (57). Because no final conclusion has been reached on the use of the same discount rate for costs and benefits, on whether the discount rate should be constant or variable over time, and on the value of the discount rate itself $(57,58)$, we recommend that the national guidelines of each country be followed.

\section{SUMMARY}

The clinical use of PET has been demonstrated to be costeffective for staging of non-small cell lung cancer, differential diagnosis of solitary pulmonary nodules, restaging of Hodgkin disease and non-Hodgkin lymphoma, and restaging of colorectal carcinoma. From a health economic point of view, the use of functional PET in clinical routine seems justified. For many other clinical indications such as monitoring response to therapy or radiation treatment planning, diagnostic effectiveness but not cost-effectiveness has been demonstrated. PET and PET/CT represent highly sensitive diagnostic tests to screen for metastatic tumor deposits in the entire body that may be missed by standard imaging modalities. The sensitivity for detection of lymph node metastases varies significantly among cancers and may be inferior compared with other techniques such as sentinel lymph node biopsy. PET/CT technology was introduced to clinical medicine in 2001, and its influence on therapeutic management has not been evaluated in detail. Moreover, clinical scenarios in which this modality can be implemented cost-effectively have not been defined yet. Initial studies have shown at least mild improvement of diagnostic accuracy, compared with separately performed CT and PET studies. Therefore, results obtained for PET can generally be extended to the PET/CT approach. There is a need for prospective, randomized clinical trials comprising high patient numbers to evaluate the clinical relevance and costeffectiveness of PET and PET/CT in other cancers such as breast cancer, melanoma, esophageal or gastric cancer, head and neck cancer, and bone and soft-tissue sarcomas. In addition, researchers may consider including cost-effectiveness studies (CUA) in clinical studies because these are increasingly requested by decision makers. 


\section{REFERENCES}

1. Juweid ME, Cheson BD. Positron-emission tomography and assessment of cancer therapy. $N$ Engl J Med. 2006;354:496-507.

2. Juweid ME, Stroobants S, Hoekstra OS, et al. Use of positron emission tomography for response assessment of lymphoma: consensus of the Imaging Subcommittee of International Harmonization Project in Lymphoma. J Clin Oncol. 2007;25:571-578.

3. Lardinois D, Weder W, Hany TF, et al. Staging of non-small-cell lung cancer with integrated positron-emission tomography and computed tomography. $\mathrm{N}$ Engl J Med. 2003;348:2500-2507.

4. Seam P, Juweid ME, Cheson BD. The role of FDG-PET scans in patients with lymphoma. Blood. 2007;110:3507-3516.

5. von Schulthess GK, Steinert HC, Hany TF. Integrated PET/CT: current applications and future directions. Radiology. 2006;238:405-422.

6. Weber WA. Positron emission tomography as an imaging biomarker. J Clin Oncol. 2006;24:3282-3292.

7. Hillner BE, Liu D, Coleman RE, et al. The National Oncologic PET Registry (NOPR): design and analysis plan. J Nucl Med. 2007;48:1901-1908.

8. Hillner BE, Siegel BA, Liu D, et al. Impact of positron emission tomography/ computed tomography and positron emission tomography (PET) alone on expected management of patients with cancer: initial results from the National Oncologic PET Registry. J Clin Oncol. 2008;26:2155-2161.

9. Hillner BE, Siegel BA, Shields AF, et al. Relationship between cancer type and impact of PET and PET/CT on intended management: findings of the national oncologic PET registry. J Nucl Med. 2008;49:1928-1935.

10. Abschlussbericht des Gemeinsamen Bundesausschusses nach $\S 91$ Abs. 7 SGB V "Krankenhausbehandlung." Bundesanzeiger. 2006;43:1374.

11. Bury T, Dowlati A, Paulus P, et al. Evaluation of the solitary pulmonary nodule by positron emission tomography imaging. Eur Respir J. 1996;9:410-414.

12. Gupta N, Gill H, Graeber G, Bishop H, Hurst J, Stephens T. Dynamic positron emission tomography with F-18 fluorodeoxyglucose imaging in differentiation of benign from malignant lung/mediastinal lesions. Chest. 1998;114:1105-1111.

13. Patz EF Jr, Connolly J, Herndon J. Prognostic value of thoracic FDG PET imaging after treatment for non-small cell lung cancer. AJR. 2000;174:769-774.

14. Fletcher JW, Djulbegovic B, Soares HP, et al. Recommendations on the use of ${ }^{18}$ F-FDG PET in oncology. J Nucl Med. 2008;49:480-508.

15. Pieterman RM, van Putten JW, Meuzelaar JJ, et al. Preoperative staging of nonsmall-cell lung cancer with positron-emission tomography. $N$ Engl J Med. 2000;343:254-261.

16. Sachs S, Bilfinger TV. The impact of positron emission tomography on clinical decision making in a university-based multidisciplinary lung cancer practice. Chest. 2005;128:698-703.

17. Berghmans T, Dusart M, Paesmans M, et al. Primary tumor standardized uptake value (SUVmax) measured on fluorodeoxyglucose positron emission tomography (FDG-PET) is of prognostic value for survival in non-small cell lung cancer (NSCLC): a systematic review and meta-analysis (MA) by the European Lung Cancer Working Party for the IASLC Lung Cancer Staging Project. J Thorac Oncol. 2008;3:6-12.

18. Hutchings M, Loft A, Hansen M, et al. FDG-PET after two cycles of chemotherapy predicts treatment failure and progression-free survival in Hodgkin lymphoma. Blood. 2006;107:52-59.

19. Weber WA. Use of PET for monitoring cancer therapy and for predicting outcome. J Nucl Med. 2005;46:983-995.

20. Ott K, Herrmann K, Lordick F, et al. Early metabolic response evaluation by fluorine-18 fluorodeoxyglucose positron emission tomography allows in vivo testing of chemosensitivity in gastric cancer: long-term results of a prospective study. Clin Cancer Res. 2008;14:2012-2018.

21. Lordick F, Ott K, Krause BJ, et al. PET to assess early metabolic response and to guide treatment of adenocarcinoma of the oesophagogastric junction: the MUNICON phase II trial. Lancet Oncol. 2007;8:797-805.

22. Ciernik IF, Dizendorf E, Baumert BG, et al. Radiation treatment planning with an integrated positron emission and computer tomography (PET/CT): a feasibility study. Int J Radiat Oncol Biol Phys. 2003;57:853-863.

23. Grosu AL, Molls M, Zimmermann FB, et al. High-precision radiation therapy with integrated biological imaging and tumor monitoring: evolution of the Munich concept and future research options. Strahlenther Onkol. 2006;182:361-368.

24. Mac Manus MP, Hicks RJ, Ball DL, et al. F-18 fluorodeoxyglucose positron emission tomography staging in radical radiotherapy candidates with nonsmall cell lung carcinoma: powerful correlation with survival and high impact on treatment. Cancer. 2001;92:886-895.

25. Facey K, Bradbury I, Laking G, Payne E. Overview of the clinical effectiveness of positron emission tomography imaging in selected cancers. Health Technol Assess. 2007;11:iii-iv, xi-267.
26. Drummond M, Sculpher M, Torrance G, O'Brien B, Stoddart G. Methods for the Economic Evaluation of Health Care Programmes. Oxford, U.K.: Oxford University Press; 2005.

27. National Institute for Health and Clinical Excellence. Guide to the Methods of Technology Appraisal. Issue date: June 2008. Available at: http://www.nice.org. uk/media/B52/A7/TAMethodsGuideUpdatedJune2008.pdf. Accessed December 8, 2009.

28. Valk PE, Pounds TR, Tesar RD, Hopkins DM, Haseman MK. Costeffectiveness of PET imaging in clinical oncology. Nucl Med Biol. 1996; 23:737-743.

29. Gambhir SS, Hoh CK, Phelps ME, Madar I, Maddahi J. Decision tree sensitivity analysis for cost-effectiveness of FDG-PET in the staging and management of non-small-cell lung carcinoma. J Nucl Med. 1996;37:1428-1436.

30. Miles KA. An approach to demonstrating cost-effectiveness of diagnostic imaging modalities in Australia illustrated by positron emission tomography. Australas Radiol. 2001;45:9-18.

31. Keith CJ, Miles KA, Griffiths MR, Wong D, Pitman AG, Hicks RJ. Solitary pulmonary nodules: accuracy and cost-effectiveness of sodium iodide FDG-PET using Australian data. Eur J Nucl Med Mol Imaging. 2002;29:1016-1023.

32. Kosuda S, Ichihara K, Watanabe M, Kobayashi H, Kusano S. Decision-tree sensitivity analysis for cost-effectiveness of chest 2-fluoro-2-D- $\left[{ }^{18} \mathrm{~F}\right]$ fluorodeoxyglucose positron emission tomography in patients with pulmonary nodules (non-small cell lung carcinoma) in Japan. Chest. 2000;117:346-353.

33. Dietlein M, Weber K, Gandjour A, et al. Cost-effectiveness of FDG-PET for the management of potentially operable non-small cell lung cancer: priority for a PETbased strategy after nodal-negative CT results. Eur J Nucl Med. 2000;27:15981609.

34. Kelly RF, Tran T, Holmstrom A, Murar JE, Segurola RJ Jr. Accuracy and costeffectiveness of $\left[{ }^{18} \mathrm{~F}\right]$-2-fluoro-deoxy-D-glucose-positron emission tomography scan in potentially resectable non-small cell lung cancer. Chest. 2004;125:14131423.

35. van Tinteren H, Hoekstra OS, Smit EF, et al. Effectiveness of positron emission tomography in the preoperative assessment of patients with suspected non-smallcell lung cancer: the PLUS multicentre randomised trial. Lancet. 2002;359:13881393.

36. Scott WJ, Shepherd J, Gambhir SS. Cost-effectiveness of FDG-PET for staging non-small cell lung cancer: a decision analysis. Ann Thorac Surg. 1998;66:18761883 .

37. Silvestri GA, Gould MK, Margolis ML, et al. Noninvasive staging of non-small cell lung cancer: ACCP evidenced-based clinical practice guidelines (2nd edition). Chest. 2007;132(3 suppl):178S-201S.

38. Park KC, Schwimmer J, Shepherd JE, et al. Decision analysis for the cost-effective management of recurrent colorectal cancer. Ann Surg. 2001;233:310-319.

39. Lejeune $\mathrm{C}$, Bismuth MJ, Conroy $\mathrm{T}$, et al. Use of a decision analysis model to assess the cost-effectiveness of ${ }^{18} \mathrm{~F}$-FDG PET in the management of metachronous liver metastases of colorectal cancer. J Nucl Med. 2005;46:2020-2028.

40. Gambhir SS, Valk P, Shepherd JE, Hoh CK, Allen M, Phelps ME. Cost-effective analysis modeling of the role of FDG PET in the management of patients with recurrent colorectal cancer [abstract]. J Nucl Med. 1997;38:90-91.

41. Hollenbeak CS, Lowe VJ, Stack BC Jr. The cost-effectiveness of fluorodeoxyglucose 18-F positron emission tomography in the N0 neck. Cancer. 2001;92: 2341-2348.

42. Hoh CK, Glaspy J, Rosen P, et al. Whole-body FDG-PET imaging for staging of Hodgkin's disease and lymphoma. J Nucl Med. 1997;38:343-348.

43. Klose T, Leidl R, Buchmann I, Brambs HJ, Reske SN. Primary staging of lymphomas: cost-effectiveness of FDG-PET versus computed tomography. Eur J Nucl Med. 2000;27:1457-1464.

44. Heinrich S, Goerres GW, Schafer M, et al. Positron emission tomography/ computed tomography influences on the management of resectable pancreatic cancer and its cost-effectiveness. Ann Surg. 2005;242:235-243.

45. Adler LP, Faulhaber PF, Schnur KC, Al-Kasi NL, Shenk RR. Axillary lymph node metastases: screening with [F-18]2-deoxy-2-fluoro-D-glucose (FDG) PET. Radiology. 1997;203:323-327.

46. Dooms CA, Lievens YN, Vansteenkiste JF. Cost-utility analysis of chemotherapy in symptomatic advanced nonsmall cell lung cancer. Eur Respir J. 2006;27:895-901.

47. Fossella F, Pereira JR, von Pawel J, et al. Randomized, multinational, phase III study of docetaxel plus platinum combinations versus vinorelbine plus cisplatin for advanced non-small-cell lung cancer: the TAX 326 study group. J Clin Oncol. 2003;21:3016-3024.

48. Miller FG, Brody H. What makes placebo-controlled trials unethical? Am J Bioeth. 2002;2:3-9.

49. Bradbury I, Bonell E, Boynton J, et al. Positron Emission Tomography (PET) Imaging in Cancer Management. Glasgow, U.K.: Health Technology Board for Scotland; 2002. 
50. Temple R, Ellenberg SS. Placebo-controlled trials and active-control trials in the evaluation of new treatments. Part 1: ethical and scientific issues. Ann Intern Med. 2000;133:455-463.

51. Institute for Quality and Efficiency in Health Care. Methods for Assessment of the Relation of Benefits to Costs in the German Statutory Health Care System. Version 1.1. Available at: http://www.iqwig.de/download/08-10-14_Methods_ of_the_Relation_of_Benefits_to_Costs_v_1_1.pdf. Accessed December 8, 2009.

52. Hollen PJ, Gralla RJ, Kris MG, Potanovich LM. Quality of life assessment in individuals with lung cancer: testing the Lung Cancer Symptom Scale (LCSS). Eur J Cancer. 1993;29A(suppl 1):S51-S58.

53. Hollen PJ, Gralla RJ, Kris MG, McCoy S, Donaldson GW, Moinpour CM. A comparison of visual analogue and numerical rating scale formats for the Lung Cancer Symptom Scale (LCSS): does format affect patient ratings of symptoms and quality of life? Qual Life Res. 2005;14:837-847.

54. Brooks R. EuroQol: the current state of play. Health Policy. 1996;37:53-72.
55. Doyle S, Lloyd A, Walker M. Health state utility scores in advanced non-small cell lung cancer. Lung Cancer. 2008;62:374-380.

56. Bird A, Norman R, Goodall S. Economic Evaluation of Positron Emission Tomography (PET) in Non Small Cell Lung Cancer (NSCLC): CHERE Working Paper 2007/6. Available at: http://ideas.repec.org/p/her/chewps/2007-6.html. Accessed December 8, 2009.

57. Severens JL, Milne RJ. Discounting health outcomes in economic evaluation: the ongoing debate. Value Health. 2004;7:397-401.

58. Bos JM, Postma MJ, Annemans L. Discounting health effects in pharmacoeconomic evaluations: current controversies. Pharmacoeconomics. 2005;23:639-649.

59. Medicare National Coverage Determinations Manual. Available at: http:// www.cms.hhs.gov/manuals/downloads/ncd103c1_Part4.pdf. Accessed December 8, 2009. Publication 100-03, Part 4.

60. Federal Joint Committee ("Gemeinsamer Bundesausschuss" [G-BA]). Bekanntmachung eines Beschlusses des Gemeinsamen Bundesausschusses Qualitätssicherungsvereinbarung PET. Bundesanzeiger. 2007;97:5382. 